\title{
Reflexo do período de lactação na produtividade de porcas primíparas e multíparas
}

\author{
Lactation lenght reflex in the productivity of the primiparous and multiparous sows
}

\author{
Fabiano Bonfim Carregaro', Ana Paula Gonçalves Mellagi', \\ Mari Lourdes Bernardi'2, Ivo Wentz' \& Fernando Pandolfo Bortolozzo'
}

\begin{abstract}
RESUMO
O período de lactação é um importante aspecto na produtividade de granjas suínas, já que influencia diretamente os dias do ciclo produtivo e a fisiologia da reprodução das matrizes. $\mathrm{O}$ objetivo deste trabalho foi verificar o efeito do período de lactação (PL) no intervalo desmame-estro (IDE), taxa de parição ajustada (TPA) e tamanho da leitegada subseqüente (TLS) em um rebanho comercial. Dados referentes a 19.846 desmames realizados em 2002 e 2003 foram coletados a partir do programa de gerenciamento de dados PigCHAMP ${ }^{\circledR}$. Para multíparas, PL de 15 dias foram suficientes para a estabilização do IDE (4,8 $\pm 2,8$ dias). As primíparas apresentaram IDE mais curto com PL de 20-24 dias (4,7 $\pm 2,5$ dias). Houve redução na TPA somente em PL inferiores a 10 dias, tanto em primíparas como em multíparas. Para primíparas, períodos de lactação a partir de 17 dias resultaram em estabilização do TLS. As porcas multíparas apresentaram aumento gradativo do TLS até 15-16 dias de lactação, quando houve estabilização. A duração da lactação influencia o desempenho reprodutivo subseqüente em primíparas e multíparas suínas. Períodos de lactação de duas semanas podem ser adequados para multíparas, mas três semanas seriam necessárias para melhor desempenho das primíparas.
\end{abstract}

Descritores: período de lactação, lactação, suíno, reprodução, taxa de parto, tamanho da leitegada.

\begin{abstract}
The lactation length is an important aspect on the productive of the swine units, since it influences directly the days of productive cycle and the reproductive physiology of the sows. The goal of this study was to determine if the length of lactation (LL) has an effect on the weaning to estrus interval (WEI), adjusted farrowing rate (AFR) and subsequent litter size (SLS) in commercial herds. Data concerning 19,846 weanings on the period 2002-2003 were obtained from a backup of the PigCHAMP ${ }^{\circledR}$ farm management program. In multiparous sows, LL of 15 days was enough to the stabilization of WEI ( $4.8 \pm 2.8$ days). The primiparous showed shorter WEI with LL of 20-24 days ( $4.7 \pm 2.5$ days). The AFR was reduced only in LL less than 10 days, in both primiparous and multiparous sows. For primiparous sows, LL more than 17 days resulted in the stabilization of SLS. In multiparous sows there was a gradative increase in SLS until 15-16 days of lactation when the SLS stabilized. The lactation length influenced the reproductive performance in primiparous and multiparous sows. Lactation lengths of two weeks may be adequate to multiparous, but three weeks are necessary to better performance of primiparous sows.
\end{abstract}

Key words: lactation length, lactation, swine, reproduction, farrowing rate, litter size. 


\section{INTRODUÇÃO}

Vários fatores influenciam o número de leitões desmamados/fêmea/ano em granjas tecnificadas. Em situações práticas, pode-se atuar em dois índices importantes. Um deles envolve o número de leitões nascidos vivos/fêmea/ano, e outro o número de partos/ fêmea/ano, o qual é influenciado diretamente pela duração da gestação, duração de lactação e dias não produtivos [4]. Algumas dessas características são constantes ou de difícil manipulação, sendo menos flexíveis nos ganhos de produtividade, como é o caso da duração da gestação. Portanto, para implementar um aumento no número de partos por matriz ao ano é necessário promover alterações na duração da lactação ou na redução dos dias não-produtivos.

O período de lactação é uma importante variável, já que, além de proporcionar alterações diretas no número de partos/fêmea/ano, também é relacionada ao nível produtivo do plantel, como demonstrado por alguns autores [3,10]. Durante a lactação, a fêmea encontra-se em anestro lactacional ou fisiológico, sendo este aspecto rapidamente revertido após o desmame [1].

Vários períodos de lactação são empregados visando a maximização dos lucros ou o bem-estar animal. Para os americanos, períodos de lactação médios em 14 dias são utilizados, pois permitem um maior número de ciclos/fêmea/ano [5]. Os europeus praticam desmames em torno de 28 dias, o que levaria o plantel a melhores índices reprodutivos [9], porém menor número de ciclos/fêmea/ano. Em uma unidade que produz 27 leitões desmamados por fêmea ao ano, cada dia representa 0,074 leitão produzido, ou seja, é fundamental encontrar um ponto de equilíbrio ideal para a duração do período lactacional que não comprometa os índices produtivos.

O objetivo deste trabalho foi verificar o efeito da duração do período de lactação (PL) no intervalo desmame-estro (IDE), taxa de parição ajustada (TPA) e tamanho da leitegada subseqüente (TLS) de fêmeas em rebanho comercial.

\section{MATERIAIS E MÉTODOS}

Os dados das fêmeas de ordem de parto 1 a 8 , referentes a um período de 2 anos (2002-2003), foram coletados a partir do programa de gerenciamento de dados PigCHAMP ${ }^{\circledR}$ de uma granja produtora de suínos (reprodutoras da linhagem Camborough $22^{\circledR}$ ), localizada na região Centro-Oeste do Brasil.
Foram analisados dados referentes à duração da lactação de 1 a 24 dias e intervalo desmame-estro menor que 16 dias. Para a análise do efeito do PL sobre IDE foram considerados dados de 19.846 desmames. Para taxa de parição ajustada e tamanho de leitegada foram analisados 16.405 e 14.309 dados, respectivamente, sendo estes somente de coberturas efetuadas no primeiro estro após o desmame. As coberturas que resultaram em alguma interrupção no ciclo produtivo, tais como retorno ao estro ou aborto, não foram incluídas para evitar qualquer influência do efeito de um período de interrupção sobre a parição e tamanho de leitegada com o efeito da duração da lactação propriamente dita.

As fêmeas foram agrupadas conforme o período de lactação em 6 classes: 1) PL de 1 a 9 dias; 2) PL de 10 a 14 dias; 3) PL de 15 a 16 dias; 4) PL de 17 dias; 5) PL de 18 a 19 dias; e 6) PL de 20 a 24 dias. A frequiência de distribuição, de acordo com o período de lactação, foi obtida pelo procedimento FREQ do SAS [12]. A TPA foi comparada, entre as classes de PL, pelo teste qui-quadrado. O IDE e TLS foram analisados pelo procedimento GLM do SAS [12], sendo as médias comparadas pelo teste de Tukey-Kramer.

\section{RESULTADOS}

A distribuição dos períodos de lactação, de acordo com sua duração, é apresentada na Figura 1. Houve concentração de 74,5\% das lactações em 16 a 18 dias, com média de $16,6 \pm 2,5$ dias.

A média geral de intervalo desmame-estro foi $5,0 \pm 2,7$ dias. Tanto em primíparas como em multíparas, o IDE diminuiu com o aumento no período de lactação (Tabela 1). Lactações com duração inferior a 10 dias resultaram em IDE igual ou superior a sete dias, em ambas as categorias. Para multíparas, houve estabilização da duração do IDE, nas lactações com 15 ou mais dias. As fêmeas primíparas apresentaram menor IDE com períodos de lactação de 20 a 24 dias.

A TPA geral foi $88,1 \%$. Houve prejuízo na TPA somente em períodos de lactação inferiores a 10 dias, tanto em primíparas como em multíparas (Tabela 1). O número médio geral do total de leitões nascidos foi $11,3 \pm 3,3$. Ocorreu aumento gradativo do TLS conforme o avanço da duração da lactação anterior (Tabela 1). Para primíparas, períodos de lactação a partir de 17 dias resultaram em estabilização do TLS. As multíparas apresentaram aumento gradativo do TLS até 15-16 dias de lactação, quando houve estabilização. 


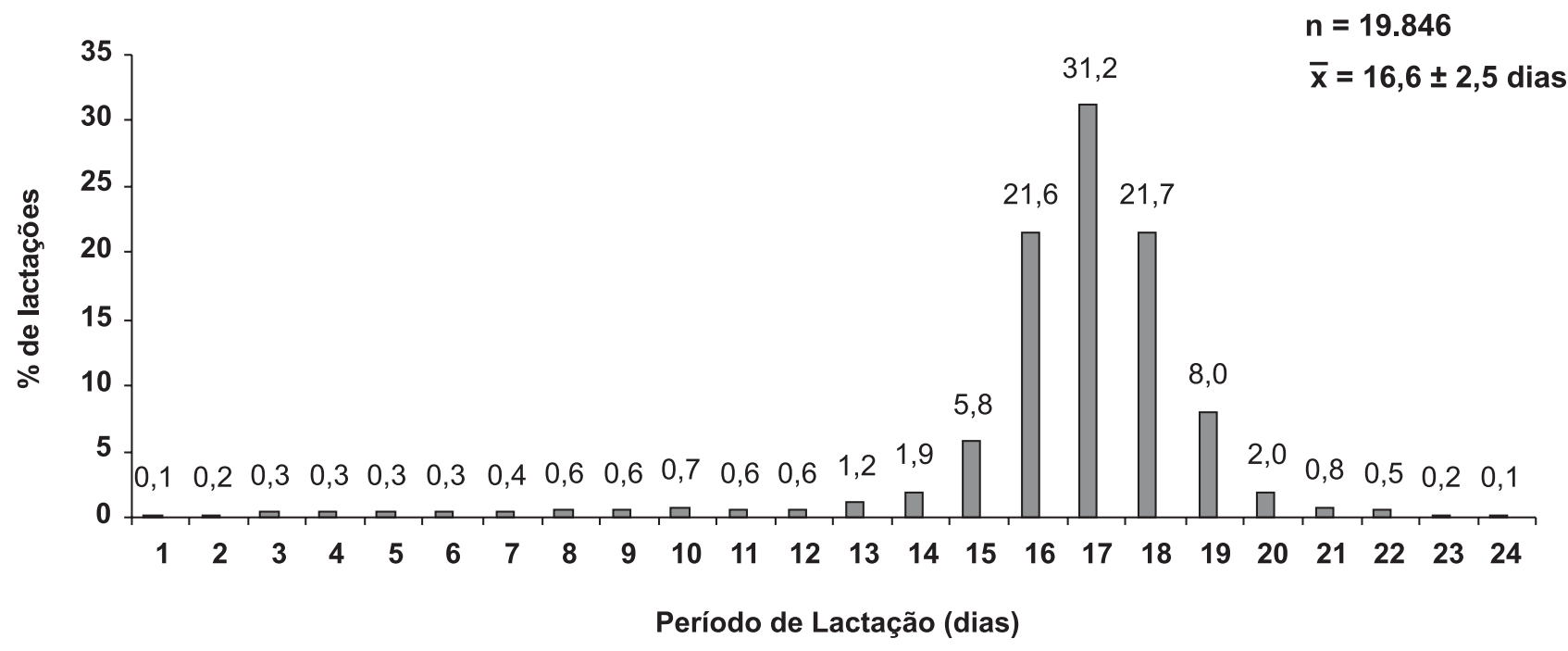

Figura 1. Frequência da distribuição das lactações de acordo com sua duração.

\begin{tabular}{|c|c|c|c|c|c|c|c|c|}
\hline & & \multirow{2}{*}{ № } & \multicolumn{6}{|c|}{ Período de Lactação (dias) } \\
\hline & & & $1-9$ & $10-14$ & $15-16$ & 17 & $18-19$ & $20-24$ \\
\hline \multirow{3}{*}{$\begin{array}{l}\text { IDE } \\
\text { (dias) }\end{array}$} & Primíparas & 3.920 & $7,0 \pm 3,8 a$ & $5,8 \pm 2,7 b$ & $5,7 \pm 2,9 b$ & $5,6 \pm 2,8 b$ & $5,5 \pm 2,9 b$ & $4,7 \pm 2,5 c$ \\
\hline & Multíparas & 15.926 & $7,1 \pm 3,2 a$ & $5,4 \pm 2,8 b$ & $4,8 \pm 2,6 c$ & $4,8 \pm 2,7 c$ & $4,8 \pm 2,6 c$ & $4,4 \pm 2,7 c$ \\
\hline & Total & 19.846 & $7,1 \pm 3,3 a$ & $5,5 \pm 2,8 b$ & $4,9 \pm 2,6 c$ & $5,0 \pm 2,7 \mathrm{c}$ & $5,0 \pm 2,7 c$ & $4,6 \pm 2,6 d$ \\
\hline \multirow{3}{*}{$\begin{array}{l}\text { TPA } \\
(\%)\end{array}$} & Primíparas & 3.056 & $68,1 \mathrm{a}$ & $87,8 b$ & $86,2 b$ & $86,1 b$ & $83,5 b$ & $82,7 b$ \\
\hline & Multíparas & 13.349 & $75,6 a$ & $87,9 b$ & $89,5 b$ & $89,3 b$ & $89,2 b$ & $90,3 b$ \\
\hline & Total & 16.405 & $74,4 a$ & $87,9 b c$ & $89,0 b$ & $88,7 \mathrm{bc}$ & $88,0 \mathrm{bc}$ & $85,8 \mathrm{c}$ \\
\hline \multirow{3}{*}{$\begin{array}{l}\text { TLS } \\
\left(\mathrm{n}^{\circ}\right)\end{array}$} & Primíparas & 2.555 & $9,3 \pm 3,5 a$ & $9,4 \pm 3,1 a$ & $10,4 \pm 3,1 \mathrm{ab}$ & $10,5 \pm 3,1 b$ & $10,6 \pm 3,1 b$ & $10,9 \pm 3,3 b$ \\
\hline & Multíparas & 11.754 & $9,7 \pm 3,3 a$ & $10,7 \pm 3,2 b$ & $11,3 \pm 3,2 \mathrm{c}$ & $11,4 \pm 3,2 \mathrm{~cd}$ & $11,6 \pm 3,3 d$ & $11,6 \pm 3,1 \mathrm{~cd}$ \\
\hline & Total & 14.309 & $9,6 \pm 3,3 a$ & $10,6 \pm 3,2 b$ & $11,2 \pm 3,2 \mathrm{c}$ & $11,3 \pm 3,2 \mathrm{~cd}$ & $11,4 \pm 3,2 d$ & $11,2 \pm 3,2 \mathrm{~cd}$ \\
\hline
\end{tabular}

\section{DISCUSSÃO}

O período de lactação influenciou o IDE. No grupo das multíparas, períodos de lactação superiores a 14 dias foram suficientes para as fêmeas apresentarem IDE médio de 3 a 5 dias, ou seja, dentro da faixa de melhor produtividade [11]. Já as primíparas necessitam de maior período lactacional (20 a 24 dias) para proporcionar IDE médio nessa mesma faixa. Alguns autores sugerem que fêmeas de primeira parição ainda não possuem maturidade fisiológica completa (tamanho e reserva corporal), em comparação às multíparas, sendo o consumo de ração durante o período de lactação insuficiente para seu desenvolvimento e para as exigências da leitegada [9]. Desta forma, há prejuízo de seu desempenho reprodutivo posterior.

No presente estudo, tanto em primíparas quanto em multíparas a taxa de parição foi influenciada apenas em desmames abaixo dos 10 dias pós-parto. Estes animais podem estar representando um grupo de fêmeas com problemas já que, possivelmente, são desmames emergenciais. Fêmeas com períodos de lactação nesta faixa, além de possíveis complicações préexistentes (problemas locomotores, disgalaxia ou alterações ao parto) são prejudicadas também fisiologicamente, pois períodos curtos de lactação não permitem o restabelecimento à normalidade uterina e ovariana. Com isso, há redução nos níveis produtivos por inse- 
minações indevidas ou apenas aproveitamento de fêmeas no segundo estro (ciclo) pós-desmame, o que influencia negativamente os dias não produtivos do plantel. Além disso, a probabilidade de desenvolvimento de cistos ovarianos em fêmeas com desmame abaixo de 14 dias é 15,7 vezes maior que as desmamadas aos 19 dias [2], sendo este um importante fator na redução da fertilidade no suíno. O puerpério se caracteriza pela involução uterina e pelo restabelecimento do ciclo estral. A involução é importante para a completa regeneração do endométrio, garantindo a ligação embrio-maternal [1]. Estudos demonstram a necessidade de 2 a 3 semanas para que ocorra a involução uterina, sendo possível o sucesso da cobertura [6]. Desta forma, reduzidos intervalos entre o parto e a inseminação são caracterizados pelo aumento da mortalidade embrionária e conseqüente redução na taxa de parto [7], apesar do número médio de ovulações não ser influenciado pela duração da lactação anterior $[9,10]$.

O aumento gradativo do TLS conforme o avanço da duração da lactação anterior estabilizou-se a partir dos 17 dias de lactação nas primíparas e aos 15 dias em multíparas, o que corrobora observações prévias $[3,8]$. Períodos curtos de lactação (8-12 dias) reduzem a sobrevivência embrionária em 18,3\% em relação a períodos de 18-21 dias [9]. Desta forma, exis- te associação entre a redução da lactação e desempenho reprodutivo, geralmente com taxa de parto e tamanho de leitegada maiores em desmames superiores a 25 dias [6]. Entretanto, o número de leitegadas produzidas por fêmea por ano é otimizado quando a duração da lactação é inferior a este período. Nesse contexto, qualquer estratégia que vise a redução da duração da lactação deve ser bem avaliada para que seja preser-vada a produtividade do plantel.

É necessário utilizar período de lactação que contemple o intervalo mínimo de recuperação do trato reprodutivo e garanta um desempenho satisfatório, sem aumentar em demasia o intervalo entre partos. Considerando os resultados do presente estudo, períodos de duas semanas de lactação podem ser utilizados em multíparas, mas três semanas de lactação seriam vantajosas para primíparas.

\section{CONCLUSÕES}

A duração da lactação influencia o desempenho reprodutivo subseqüente em primíparas e multíparas suínas. Ao reduzir o período de lactação há um aumento no IDE, principalmente em primíparas. A taxa de parição ajustada é reduzida em períodos de lactação inferiores a 10 dias. Em lactações mais longas há aumento no tamanho da leitegada, estabilizandose a partir dos 17 dias de lactação nas primíparas e já aos 15 dias em multíparas.

\section{REFERÊNCIAS}

1 Anderson L.L. 1993. Pig. In: Hafez E.S.E. (Ed). Reproduction in Farm Animals. 6th edn. Philadelphia: Lea \& Febiger, pp.343-360.

2 Castagna C.D., Peixoto C.H., Bortolozzo F.P., Wentz I., Borchardt Neto G. \& Ruschel F. 2004. Ovarian cysts and their consequences on the reproductive performance of swine herds. Animal Reproduction Science. 84: 115-123.

3 Costa E.P., Amaral Filha W.S., Carvalho F.F., Santos A.K. \& Silva A.S. 2004. Influence of the lactation lenght in the subseqüent litter size in sows. Animal Reproduction. 1: 111-114.

4 Dial G.D., Marsh W.E., Polson D.D. \& Vaillancourt J.P. 1992. Reproductive Failure: differential diagnosis. In: Leman A.D., Straw B.E., Mengeling W.L., D'allaire S., Taylor D.J.(Eds). Diseases of Swine. 7th edn. Iowa: Wolfe, pp.88-137.

5 Fahmy M.H. 1981. Factors influencing the weaning to oestrus interval in swine: a review. World Reviews of Animal Production. 17: 15-28.

6 Flowers W.L. 1998. Management of reproduction. In: Wiseman J., Varley M.A. \& Chadwinck J.P. (Eds). Progress in Pig Science. Nottingham: Nottingham University Press, pp.383-405.

7 Gaustad-Aas A.H., Hofmo O.P. \& Kralberg K. 2004. The importance of farrowing to service interval in sows served during lactation or after shorter lactation than 28 days. Animal Reproduction Science. 81: 287-293.

8 Kiracofe G.H. 1980. Uterine involution: its role in regulating postpartum intervals. Journal of Animal Science. 51(Suppl 2): 16-28.

9 Koketsu Y. \& Dial G.D. 1997. Factors influencing the postweaning reproductive performance of sows on commercial farms. Theriogenology. 47: 1445-1461.

10 Marsteller T.A., Armbruster G.A., Anderson D.B., Wuethrich A.J., Taylor J.L. \& Symanowski J.T. 1997. Effect of lactation length on ovulation rate and embryo survival in swine. Swine Health and Production. 5: 49-56. 
11 Poleze E., Kummer R., Bernardi M.L., Soares J.L., Bortolozzo F.P. \& Wentz I. 2003. Consequências reprodutivas da variação do intervalo desmame-estro em suínos. In: Anais do XI Congresso Brasileiro de Veterinários Especialistas em Suínos (Goiânia, Brasil). pp.187-188.

12 SAS Institute INC. 2000. SAS User's Guide: Statistical Analysis System, Release 8.0, Cary, North Carolina, U.S.A. 